\title{
Fabriques des niveaux archéologiques : méthode et premier bilan des apports à l'étude taphonomique des sites paléolithiques
}

Fabrics analysis: method, application to palaeolithic sites, and contribution to site formation studies

\section{Pascal Bertran et Arnaud Lenoble}

\section{OpenEdition \\ Journals}

Édition électronique

URL : https://journals.openedition.org/paleo/1383

DOI : $10.4000 /$ paleo.1383

ISSN : 2101-0420

Éditeur

SAMRA

Édition imprimée

Date de publication : 1 décembre 2002

Pagination : 13-28

ISSN : 1145-3370

Référence électronique

Pascal Bertran et Arnaud Lenoble, «Fabriques des niveaux archéologiques : méthode et premier bilan des apports à l'étude taphonomique des sites paléolithiques », PALEO [En ligne], 14 | 2002, mis en ligne le 09 août 2010, consulté le 21 septembre 2021. URL : http://journals.openedition.org/paleo/1383 ; DOI : https://doi.org/10.4000/paleo.1383

Ce document a été généré automatiquement le 21 septembre 2021.

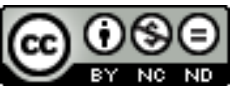

PALEO est mis à disposition selon les termes de la licence Creative Commons Attribution - Pas d'Utilisation Commerciale - Pas de Modification 4.0 International. 


\section{Fabriques des niveaux archéologiques : méthode et premier bilan des apports à l'étude taphonomique des sites paléolithiques}

Fabrics analysis: method, application to palaeolithic sites, and contribution to site formation studies

\section{Pascal Bertran et Arnaud Lenoble}

Nous remercions L. Bourguignon, L. Detrain et I. Ortega (INRAP) pour nous avoir permis d'utiliser des résultats inédits provenant de leurs fouilles respectives, ainsi que les relecteurs, J.P. Rigaud et J.P. Texier.

\section{Introduction}

De très nombreux sites paléolithiques français sont inclus dans des dépôts de versant, notamment dans les talus en pied d'abris-sous-roche, sous le porche des grottes ou dans les colluvions qui recouvrent les terrasses fluviatiles. Dans ce type d'environnement sédimentaire, les mécanismes qui conduisent à l'enfouissement d'objets déposés à la surface du sol sont complexes. Les diverses expériences réalisées dans des environnements naturels indiquent que les objets (des cailloux marqués dans la plupart des expériences) subissent des déplacements plus ou moins importants sur la pente avant d'être enfouis. Ces déplacements peuvent être très rapides (plusieurs mètres au cours d'un événement pluvieux par exemple), parfois sélectifs, c'est-à-dire qu'ils ne concernent alors que certaines catégories d'éléments, et peuvent varier considérablement sur de courtes distances en fonction des caractéristiques du sol et des singularités topographiques locales. Appliquées à l'archéologie, ces études 
démontrent que des remaniements significatifs sont susceptibles d'avoir affecté les niveaux paléolithiques ou, en d'autres termes, que les processus naturels qui se sont produits après l'abandon du site par les hommes ont pu jouer un rôle important dans la constitution des niveaux archéologiques tels qu'on les observe à l'occasion des fouilles. De manière à mettre en évidence l'impact de ces processus, il est nécessaire de déterminer en détail les mécanismes qui ont contribué à la formation du niveau archéologique étudié et de tester, à partir de la distribution spatiale et de l'organisation des vestiges, ce qui revient à la dynamique naturelle.

Différents outils sont à la disposition de l'archéologue dans cette démarche (voir par exemple Bertran et Texier 1997). Parmi ceux-ci, l'analyse des fabriques, c'est-à-dire l'analyse de l'orientation et de l'inclinaison des objets au sein du niveau archéologique, a connu d'importants développements ces dernières années concernant à la fois la méthodologie et le nombre de sites analysés. Ce type d'étude a été introduit en archéologie par Isaac (1967), Bar Yosef et Tchernov (1972) et Schick (1986) et Kaufulu (1987), initialement dans le but de comprendre la genèse des sites du Paléolithique ancien trouvés dans les dépôts alluviaux en Afrique et au Proche-Orient. Pour les dépôts de versant, l'application à l'archéologie est plus récente (Bertran 1994, Bertran et Texier 1995) et a bénéficié d'une mise au point récente sur les différents milieux dynamiques naturels (Bertran et al. 1997). Le présent article se propose de faire le point sur les progrès réalisés dans l'analyse des fabriques depuis ces derniers travaux et de montrer, à travers un certain nombre d'exemples, les potentialités de la méthode.

\section{Méthode}

3 Sur le terrain, on distingue plusieurs grands types de disposition des objets (fig. 1):

A) une disposition désordonnée (isotrope),

B) une disposition à plat sur le plan de stratification,

C) une orientation préférentielle des éléments allongés selon une direction particulière,

D) une imbrication ou disposition en tuiles, c'est-à-dire une disposition relevante par rapport au plan de stratification. 
Fig. 1 - Types de fabriques Fig. 1 - Fabric types
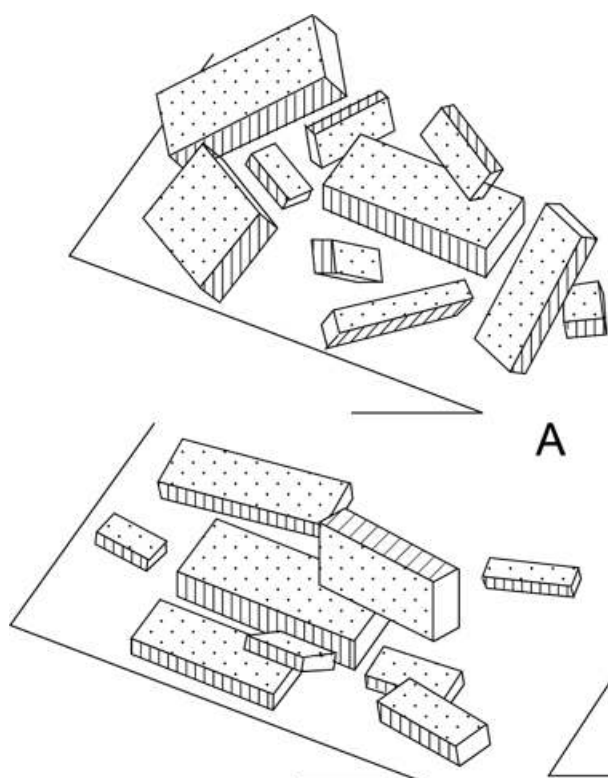

A

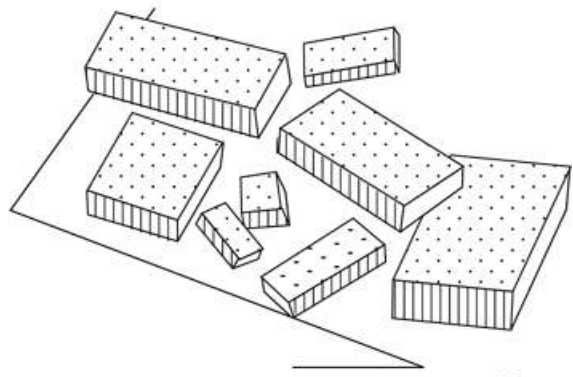

B

C

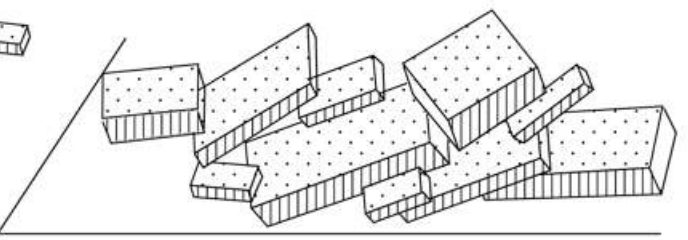

A - isotrope (désordonnée), B - planaire (à plat sur le plan de stratification), C - linéaire (orientation préférentielle du grand axe des objets), $\mathrm{D}$ - imbrication

$A$ - isotropic, $B$ - planar, $C$ - linear, $D$ - imbrication

La fabrique est quantifiée par la mesure sur un échantillon représentatif, soit une cinquantaine d'éléments, de l'orientation et de l'inclinaison du grand axe des objets. La mesure s'effectue à l'aide d'une boussole et d'un clinomètre. Les objets doivent avoir un allongement bien marqué ; dans la pratique, la limite inférieure du rapport longueur / largeur utilisé est égale à 2 .

Deux types de traitements statistiques sont utilisés pour comparer les fabriques. L'intensité de l'orientation préférentielle (Vector Magnitude) notée L est calculée selon la formule proposée par Curray (1956) :

$\mathrm{L}=100 . r . \mathrm{n}^{-1}$

avec $r=\left[(\Sigma \sin 2 \alpha)^{2}+(\Sigma \cos 2 \alpha)^{2}\right]^{0,5}$

$\mathrm{n}:$ nombre de mesures

a : orientation (de 0 à $\left.180^{\circ}\right)$

L varie de $0 \%$ (l'orientation des axes est aléatoire) à $100 \%$ (tous les axes sont orientés dans la même direction). Ce paramètre, qui n'a de réel sens que si la distribution des orientations n'est pas bi- ou plurimodale, peut être utilisé dans un test de Rayleigh pour calculer la probabilité $\mathrm{p}$ d'obtenir une valeur d'intensité plus forte que L par combinaison de $\mathrm{n}$ vecteurs d'orientation aléatoires. $\mathrm{p}$ est obtenu par la formule suivante :

$\mathrm{p}=\mathrm{e}^{(-\mathrm{L} 2 \mathrm{n})(10-4)}$

Lorsque $\mathrm{p}$ est inférieur à 0,05 , l'hypothèse d'une distribution aléatoire de l'orientation des axes des objets peut être rejetée avec un risque faible.

Les tests montrent que la valeur de L se stabilise rapidement lorsque l'échantillon atteint 40 à 50 objets mesurés (fig. 2), tandis que $p$ tend à décroître au fur et à mesure 
que $\mathrm{n}$ augmente : une orientation préférentielle non significative lorsque la taille de l'échantillon est de 50 objets peut s'avérer significative pour $n=80$. Pour un échantillon “ standard " $(n=40$ à 50$)$, la valeur de p permet de séparer les fabriques fortement linéaires $(p<0,05)$ des autres types de fabriques.

Fig. 2 - Evolution des paramètres $L$ (en haut du diagramme) et $p$ en fonction du nombre de mesures pour trois échantillons différents

Fig. 2 - Evolution of $L$ (top) and $p$ values as a function of the number of measurements for three data sets

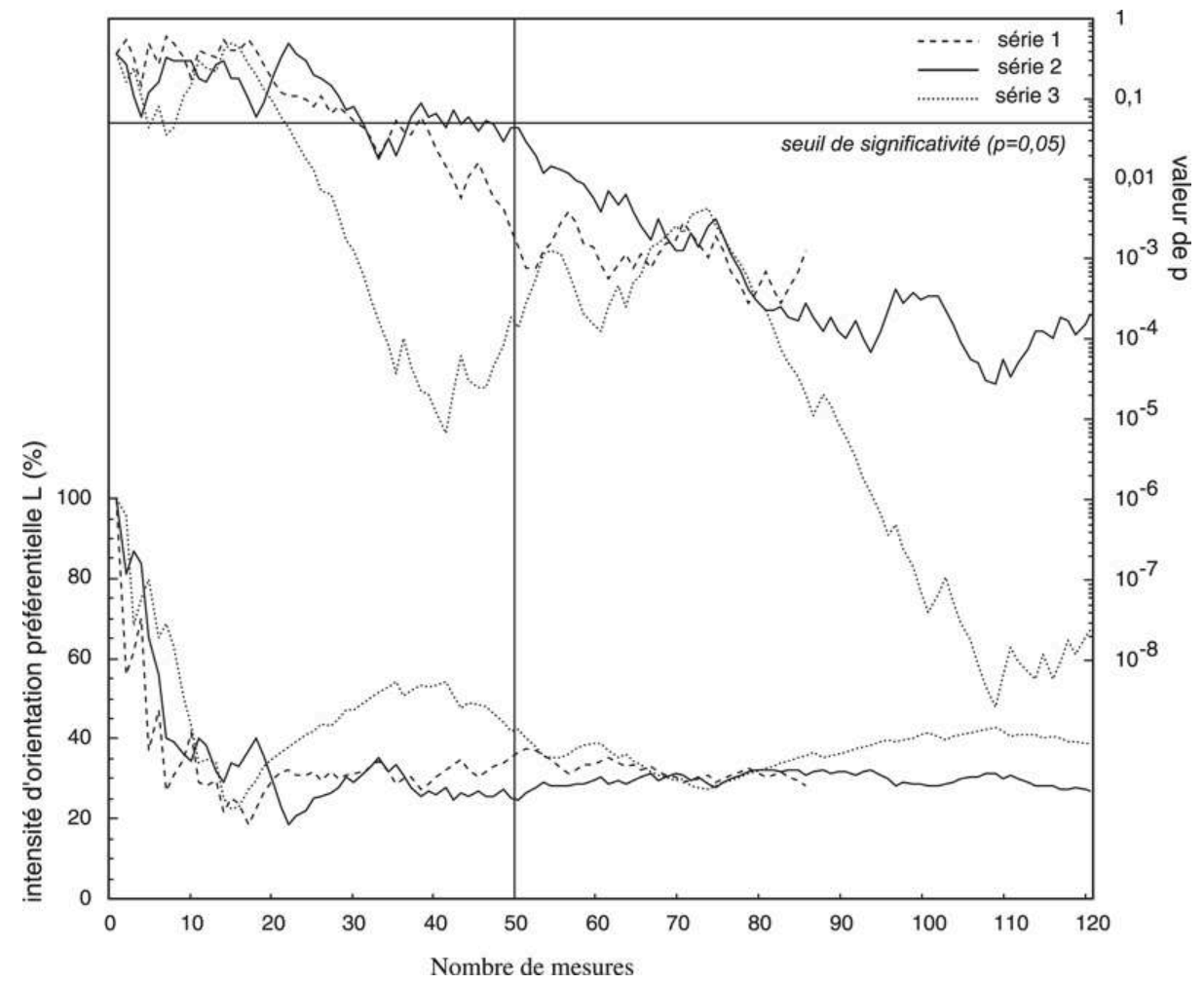

La Méthode des Valeurs Propres (Watson, 1965, 1966 ; Mark, 1973) prend en compte à la fois l'orientation et l'inclinaison des débris. L'attitude des $\mathrm{n}$ mesures peut être représentée par une matrice $3 \times 3$ construite à partir des cosinus des angles que font les axes des débris avec les trois axes de l'espace. Les vecteurs propres de la matrice définissent les axes d'étirement du nuage de points. Les valeurs propres normalisées $E_{1}$, $E_{2}$ et $E_{3}$ qui leur sont associées permettent de comparer les fabriques des différents échantillons. Pour plus de détails sur cette méthode, nous renvoyons aux publications de Watson (1965, 1966), Mark (1973) et Woodcock (1977). Le traitement des données s'effectue à l'aide d'un logiciel adapté (e.g. Stereo, Mc Eachrean, 1990). La forme de la fabrique (fabric shape) peut être caractérisée en calculant les valeurs $r_{1}=\ln \left(E_{1} / E_{2}\right), r_{2}=$ $\ln \left(E_{2} / E_{3}\right)$ et $K=r_{1} / r_{2}$. Lorsque $K$ est compris entre 0 et 1 , la fabrique est de type ceinture ou planaire (les axes des objets sont regroupés autour d'un plan, généralement le plan de stratification), entre 1 et $千$, la fabrique est de type groupé ou linéaire (les axes sont regroupés autour d'une linéation, généralement la pente). La variance sphérique (SVAR) reflète la dispersion des axes autour de l'orientation moyenne. Ce paramètre est une mesure de l'intensité de la fabrique (fabric strength), de fortes valeurs indiquant une dispersion importante des axes. Benn (1994) a également introduit un indice d'isotropie IS = E3 / E1 et un indice d'élongation EL = 1 - (E2 / E1). 
Plusieurs types de représentation sont utilisés. Lorsqu'on ne s'intéresse qu'à un seul paramètre (orientation ou inclinaison), les données sont figurées par des diagrammes en rose. Ils correspondent à des histogrammes circulaires où les valeurs sont regroupées dans des classes de 10 ou $20^{\circ}$ (fig. 3). Des classes de $20^{\circ}$ semblent être la limite admissible pour espérer mettre en évidence une orientation ou une inclinaison préférentielle des objets. Lorsque l'on dispose pour chaque objet des deux paramètres orientation et inclinaison, on utilise la projection stéréographique de Schmidt. Une description de la technique est proposée par Henry (1976). Sur le stéréogramme, les axes se matérialisent par des points, tandis que les surfaces (plan de stratification par exemple) le sont par des arcs de cercle (fig. 4).

11 Plusieurs types de diagrammes ont été proposés pour représenter les échantillons analysés par rapport aux trois pôles possibles (fabrique isotrope, fabrique planaire, fabrique linéaire). Le diagramme de Woodcock (1977) fait intervenir les paramètres $r_{1}$ et $r_{2}$ dérivés de la méthode des valeurs propres (fig. 5). Le diagramme de Benn (1994) utilise les indices IS et EL comme variables (fig. 6).

Fig. 3 - Histogramme circulaire des orientations. Exemple provenant d'un niveau moustérien du site de Baume-Vallée (Massif Central), d'après Bertran (1994)

Fig. 3 - Circular histogram of orientations. Example taken from a Mousterian level of Baume-Vallée (French Massif Central), from Bertran (1994)

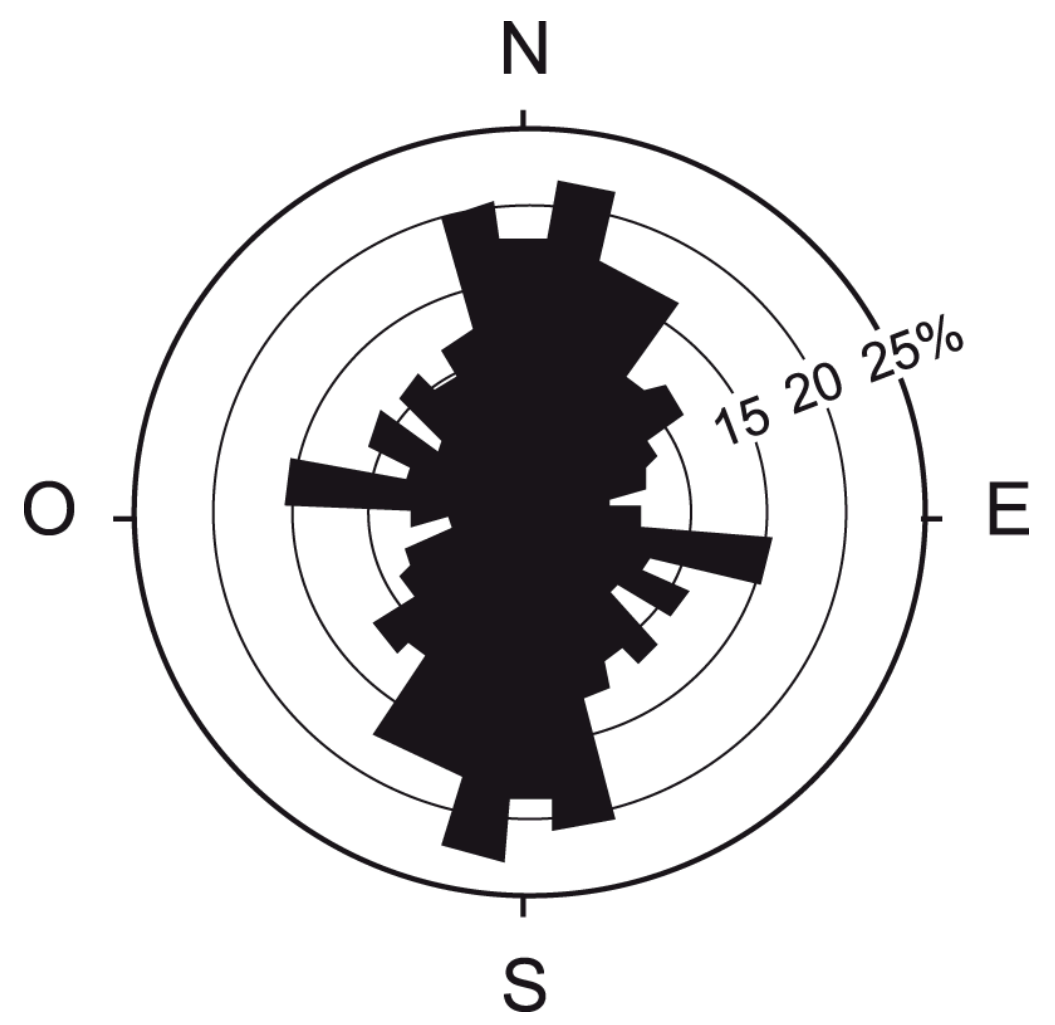


Fig. 4 - Diagrammes de Schmidt Fig. 4 - Schmidt diagrams

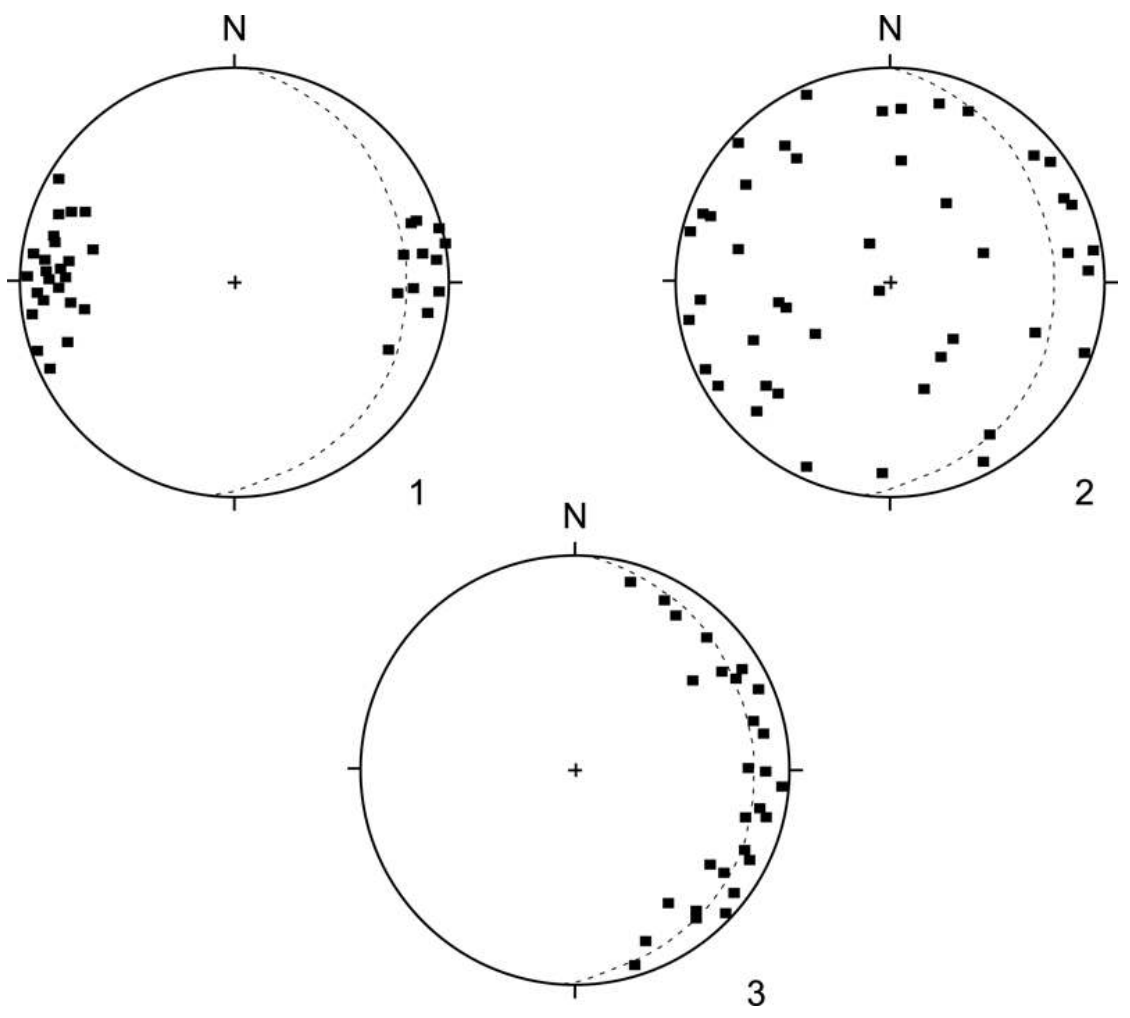

1 - fabrique linéaire (les points représentatifs des axes sont groupés autour d'une direction), 2 fabrique isotrope (les points sont dispersés), 3 - fabrique planaire (les points se regroupent sur un plan indiqué par le trait pointillé)

1 - linear fabric (dots that represent the A-axis of the artefacts are clustered), 2 - isotropic fabric (dots are scattered), 3 - planar fabric (dots are aligned along a plane indicated by the doted line) 
Fig. 5 - Diagramme de Woodcock Fig. 5 - Woodcock's diagram

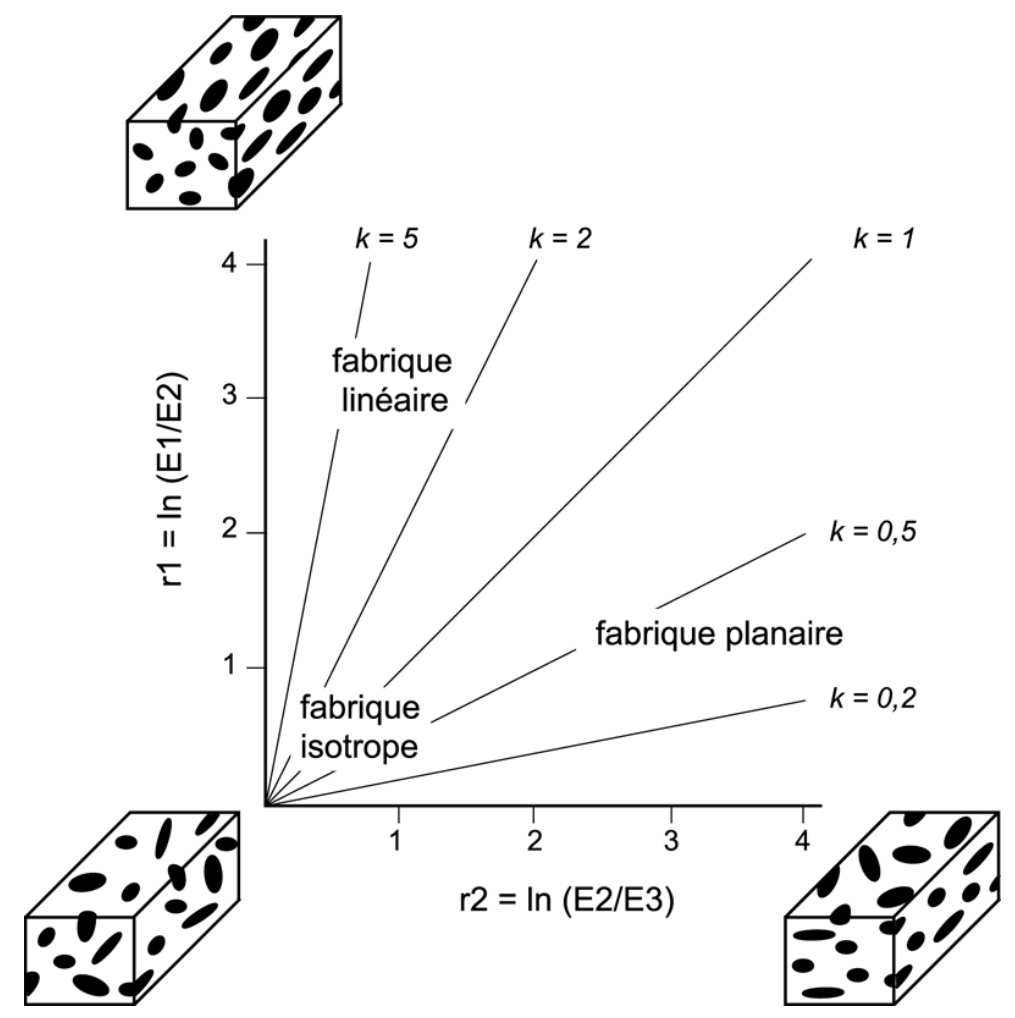

Fig. 6 - Diagramme de Benn

Fig. 6 - Benn's diagram

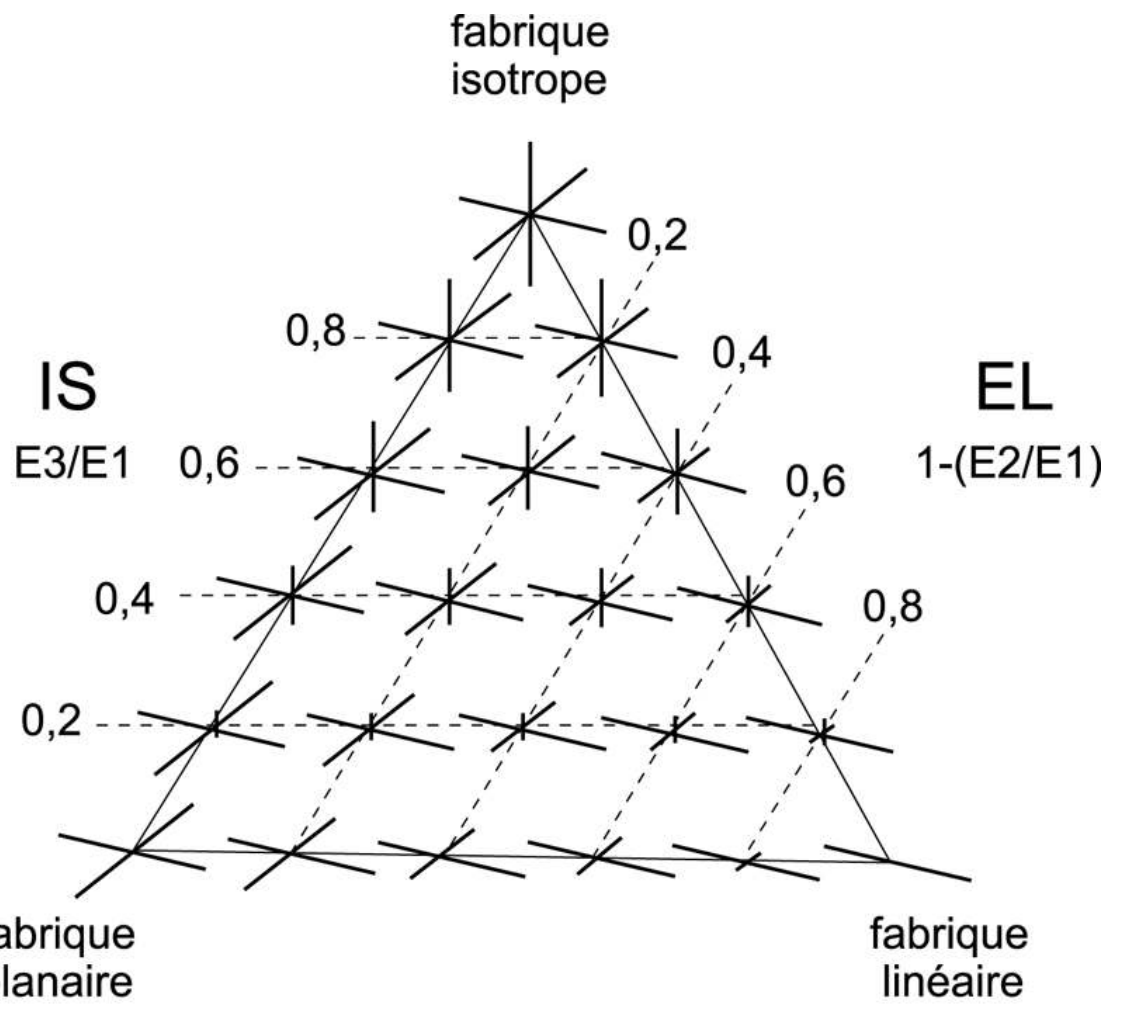




\section{Résultats}

\section{Le référentiel sur les milieux de versant actuels}

12 De nombreux travaux de géomorphologie réalisés depuis les années 1970 ont montré que des fabriques spécifiques se développent en fonction des mécanismes sédimentaires mis en jeu. Grâce à ces travaux, on dispose d'un référentiel bien documenté sur de nombreux milieux de versant. D'après Bertran et al. (1997), les fabriques permettent de distinguer deux principaux types de processus :

1. les processus de transport en masse tels que la reptation, la solifluxion, les coulées de débris et les coulées sèches, dans lesquels les éléments grossiers tendent à acquérir une orientation préférentielle dans le sens du déplacement ;

2. les processus de transport particulaire où les éléments se déplacent isolément sur le sol, tels que l'éboulisation et le ruissellement qui produisent des fabriques de type isotrope ou planaire, parfois plurimodale (fig. 7). Parmi tous les mécanismes sédimentaires analysés, la solifluxion périglaciaire donne les plus fortes valeurs d'orientation préférentielle de l'ensemble des échantillons. Les fabriques isotropes se trouvent principalement dans les éboulis gravitaires et dans les lobes des coulées de débris tandis que le ruissellement donne surtout des fabriques planaires.

Des variations importantes s'observent pour chaque processus : la figure 7 indique par exemple que selon la valeur de la pente, le ruissellement peut faire apparaître des fabriques soit de type ceinture simple, soit de type ceinture avec un mode bien marqué dans la pente. Dans le cas de pentes supérieures à $30^{\circ}$, une orientation très significative des objets apparaît. Cette étude montre également que les champs couverts par les valeurs provenant des différents processus se recouvrent de manière importante; l'analyse des fabriques ne permet donc pas, dans de nombreux cas, de discriminer les différentes dynamiques de sédimentation et doit pour cela être associée à d'autres critères. L'association possible de plusieurs dynamiques qui agissent de manière concomitante ou se succèdent dans le temps sur un même versant complique encore ce schéma. Ainsi, l'essentiel de la sédimentation (en terme de volume de matériel déposé) sur de nombreux cônes de déjection est souvent lié à des coulées de débris qui sont déclenchées par des pluies exceptionnelles. De telles coulées ont un temps de retour élevé (de cinq à plusieurs centaines d'années selon les bassins versants) et sont caractérisées par un impact morphogénétique très important. En dehors des brefs épisodes de mise en place des coulées, les dépôts du cône sont repris par d'autres dynamiques (ruissellement, solifluxion) de récurrence plus élevée et qui confèrent au dépôt final une grande partie de ses caractéristiques sédimentologiques. Les analyses montrent alors que les fabriques initiales peuvent être complètement oblitérées par la dynamique de remaniement et ne refléter que l'action du dernier phénomène intervenu sur le sédiment. 
Fig. 7 - Fabriques de dépôts de versant naturels, diagramme de Benn. D'après Bertran et al. (1997), complété

Fig. 7 - Fabric of natural slope deposits, Benn's diagram. From Bertran et al. (1997), augmented
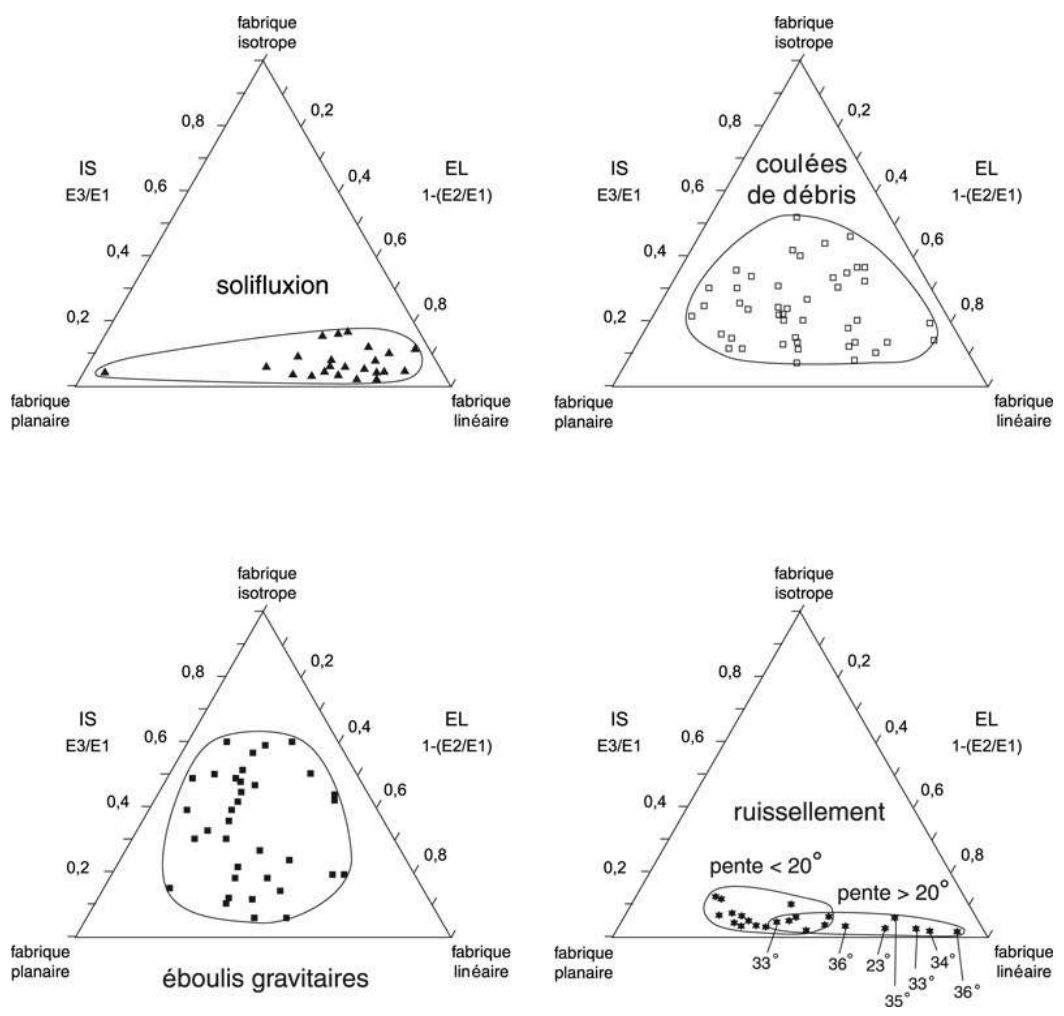

Parallèlement à ces travaux dans les milieux naturels, quelques études ont été conduites sur du matériel archéologique expérimental; elles concernent principalement la solifluxion et le ruissellement. Dans le cadre du programme TRANSIT, qui s'est déroulé sur un versant où la dynamique est dominée par la solifluxion dans le massif de la Mortice (Alpes du sud) (Texier et al. 1998, Todisco et al. 2000), différentes cellules expérimentales comportant chacune une centaine d'objets ont été disposées sur des pentes de 5 à $12^{\circ}$. Au cours de cinq ans d'expérimentation, la solifluxion a occasionné un déplacement moyen de l'ensemble des objets compris entre 4,5 et $18 \mathrm{~cm}$ vers l'aval. Quelques pièces ont toutefois subi un plus grand déplacement, de l'ordre du mètre, sous l'action d'autres processus (ruissellement, impact de la grêle, vent...). Il a été noté que l'acquisition d'une orientation préférentielle pour les éléments allongés pouvait être un phénomène relativement rapide : au cours des cinq années d'évolution à la surface d'une coulée de solifluxion, de nombreux éléments ont en effet subi un pivotement qui les a orientés dans la pente. Malgré tout, en raison de la courte durée de l'expérience, l'orientation des objets pouvait toujours être considérée comme statistiquement aléatoire.

Dans une expérience de Lenoble et Bordes (2001), des niveaux archéologiques expérimentaux ont été soumis au piétinement puis à une érosion par ruissellement diffus. Les résultats de l'expérience montrent que, sur terrain plat, le piétinement tend à engendrer une disposition plus désordonnée des objets qu'au départ, au sein d'une "couche active" de trois à cinq centimètres d'épaisseur. Lorsque la pente dépasse $10^{\circ}$, une tendance à l'acquisition d'une orientation préférentielle se manifeste en raison de la reptation progressive des objets vers l'aval au fur et à mesure du piétinement. Cette orientation reste cependant peu significative pour des échantillons de 50 objets. Au 
cours de l'érosion par le ruissellement, l'entraînement sélectif du sédiment fin provoque une diminution d'épaisseur du niveau archéologique (phénomène de pavage). La fabrique reprend alors des caractéristiques très proches de l'état initial, de type ceinture. L'observation détaillée $d u$ mode de formation du pavage indique qu'il s'accompagne du pivotement de nombreuses pièces; des orientations préférentielles parallèles ou transverses à l'écoulement se développent alors localement au sein de groupes d'objets en fonction des particularités locales. De telles observations rejoignent celles déjà faites par Butzer (1982). Cependant, à l'échelle de surfaces assez grandes, de l'ordre du mètre-carré et plus, ces phénomènes ne provoquent pas de modification sensible des paramètres de fabrique qui reste de type ceinture. L'évolution des valeurs IS et EL au cours des différentes phases de l'expérience est retracée sur la figure 8.

Fig. 8 - Evolution des paramètres IS et EL au cours de l'expérience de Lenoble et Bordes (2001)

Fig. 8 - Evolution of IS and EL values during the Lenoble and Bordes (2001) experiment.

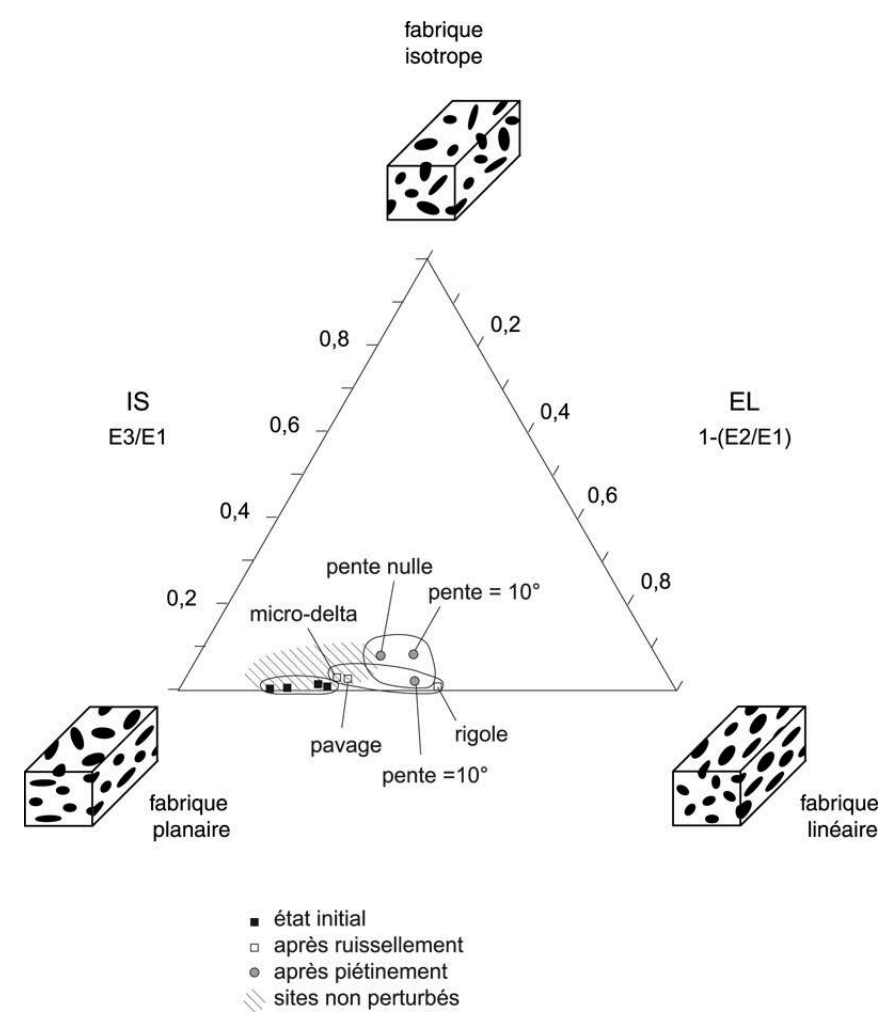

L'aire définie par les sites archéologiques non perturbés analysés dans cette étude est figurée en hachures pour comparaison

The area that corresponds to weakly disturbed sites is indicated for comparison

\section{Exemples d'application à l'archéologie}

\section{Etiolles}

Etiolles est un site madgalénien du Bassin parisien localisé sur une terrasse alluviale de la Seine (Taborin, 1994) (fig. 9). Les sols d'occupation découverts sont inclus dans des limons de débordement du fleuve et ont livré des structures d'habitat dans un état de conservation remarquable. Les résultats présentés ici concernent des mesures de fabriques effectuées en 1996 sur plusieurs concentrations de vestiges lithiques dans l'unité d'occupation D71. Ces concentrations étaient disposées sur des pentes variables, 
allant de 10 à $26^{\circ}$, correspondant à différents secteurs d'un petit chenal recoupant les dépôts alluviaux.

Fig. 9 - Carte de localisation des différents sites étudiés dans le texte

Fig. 9 - Location map of the study sites

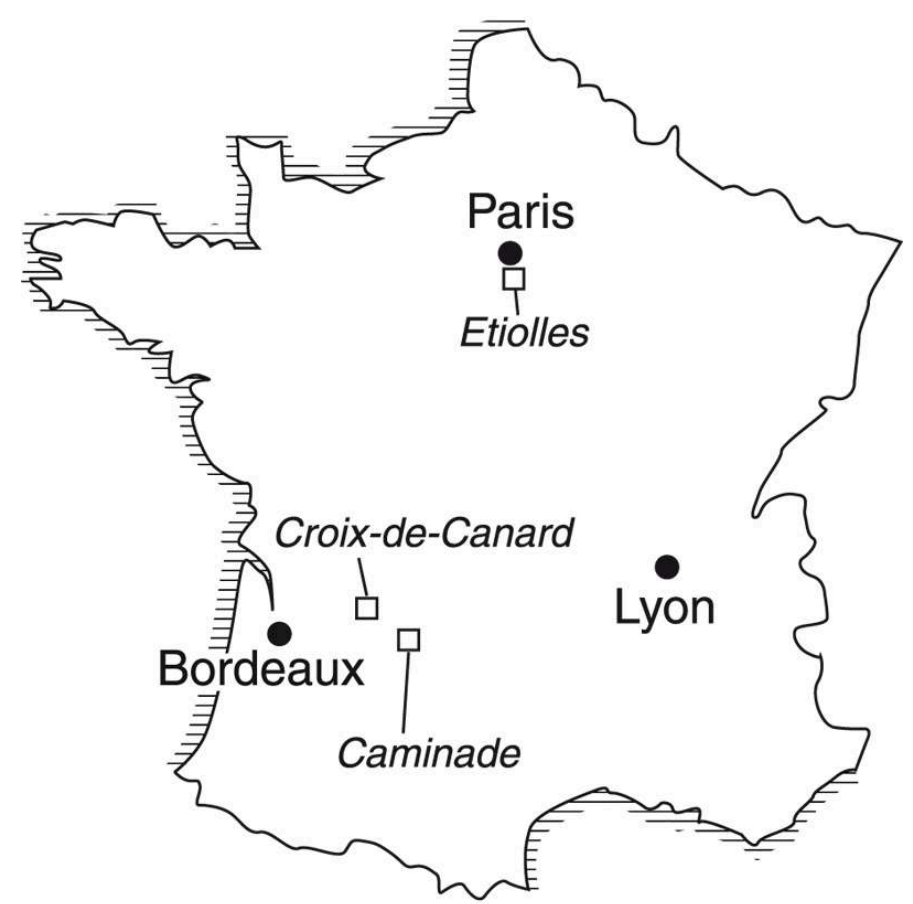

$100 \mathrm{~km}$

17 Cinq amas de débitage et un ensemble d'objets répartis sur une zone de quelques mètres carrés ont fait l'objet d'une analyse. Les fabriques se répartissent schématiquement en deux types :

- des fabriques pouvant être considérées comme planaires, avec une valeur de p supérieure à 0,05 . Ces fabriques proviennent de lots d'objets sur des pentes faibles ( 10 à $\left.17^{\circ}\right)$. Les valeurs du paramètre L, qui représente l'intensité de l'orientation préférentielle, sont faibles et comparables à celles trouvées sur d'autres sites non perturbés par Bertran et Texier (1995). Elles confirment le caractère statistiquement aléatoire de l'orientation des objets archéologiques accumulés par des processus exclusivement anthropiques ;

- des fabriques significativement orientées dans la pente $(\mathrm{p}<0,05)$. Elles correspondent aux amas de débitage localisés sur une pente forte (19 à $\left.26^{\circ}\right)$.

Dans le détail, on constate que la valeur de L est nettement corrélée avec la pente (fig. 10). Deux hypothèses peuvent être proposées pour expliquer cette observation :

la présence d'une orientation préférentielle serait d'origine primaire. On peut par exemple supposer que la position prise par les objets qui tombent sur un sol en pente au cours de la taille d'un bloc de silex ne soit pas aléatoire, les objets s'immobilisant après une phase de glissement qui peut être favorisée par la faible rugosité du substratum; cette organisation peut indiquer une réorganisation post-dépositionnelle des objets. Mills (1983) a par exemple décrit le développement d'une fabrique orientée suite à l'action d'une lente reptation des cailloux sur une pente et, de manière concomitante, 
une corrélation entre l'intensité de l'orientation préférentielle et la valeur de la pente. Dans le contexte sédimentaire du site d'Etiolles, cela pourrait résulter d'un déplacement des objets au cours des inondations qui ont déposé les sédiments limoneux. Les artefacts, trop volumineux pour être transportés par roulement ou saltation, auraient néanmoins subi des réajustements par glissement sur le sol, d'autant plus importants que leur position initiale était instable.

21 L'étude des fabriques met ici en lumière d'éventuelles perturbations passées jusqu'alors inaperçues et qui peuvent avoir des répercussions sur l'interprétation fonctionnelle de certains amas, eu égard aux possibles modifications de leur microstratification technologique. Dans l'état actuel des connaissances, il est difficile de déterminer laquelle des deux hypothèses est la plus vraisemblable; seules des expérimentations permettraient de trancher.

Fig. 10 - Corrélation entre la valeur de la pente et celle du paramètre $L$ (intensité de l'orientation préférentielle) pour le niveau d'occupation D71 du site d'Etiolles. Chaque amas analysé est représenté par un point

Fig. 10 - Correlation between slope and L value (Vector Magnitude) for the D71 occupation level of Etiolles (Paris Basin). Each dot corresponds to a debitage heap

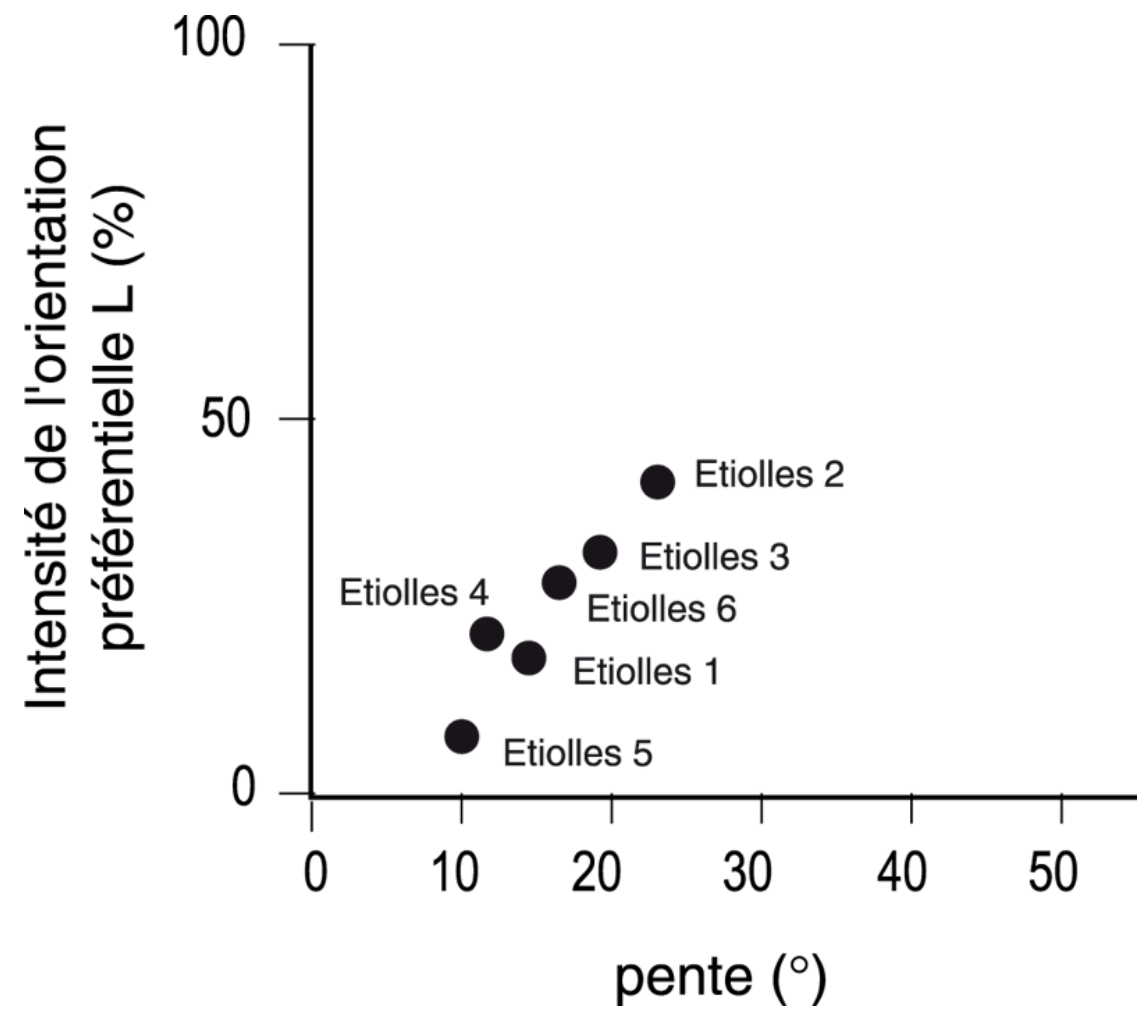

\section{Croix-de-Canard}

Le site de Croix-de-Canard, découvert à l'occasion des travaux d'archéologie préventive sur le tracé de l'autoroute A89 et fouillé par L. Detrain (INRAP), est localisé sur une terrasse de la vallée de l'Isle, à une trentaine de kilomètres à l'ouest de Périgueux (fig. 9). Plusieurs niveaux archéologiques ont été mis en évidence sur ce site. Le plus ancien ("secteur 3"), qui contient une industrie du Paléolithique moyen, est inclus dans des argiles palustres à la base de la séquence sédimentaire, à environ $4,10 \mathrm{~m}$ sous la surface actuelle du sol. Les données stratigraphiques, en particulier l'âge supposé de la 
terrasse, permettent d'attribuer un âge ancien à cette industrie, probablement de l'ordre de $300 \mathrm{ka}$. Un second niveau paléolithique moyen ("secteur 2") a été trouvé à environ $0,60 \mathrm{~m}$ de profondeur. Il est inclus dans des colluvions sablo-argileuses recouvrant les dépôts alluviaux et dont la mise en place est attribuée au dernier cycle climatique.

Dans l'ensemble des niveaux archéologiques de Croix-de-Canard, le caractère acide des sédiments est responsable de la disparition complète des vestiges osseux. A l'exception de ce type de vestiges, le contexte sédimentaire dans lequel se trouve le niveau archéologique du secteur 3 , qui correspond à des dépôts de débordement de la rivière de granulométrie très fine, a été a priori favorable à une bonne préservation du site et notamment à l'absence de déplacement latéral important: les écoulements qui ont permis l'enfouissement du niveau archéologique étaient en effet très peu puissants et incapables de déplacer des objets de taille subcentimétrique. On peut donc considérer que la distribution spatiale des vestiges est presqu'exclusivement le résultat de l'activité anthropique. Certaines observations, en particulier la présence de nombreux objets disposés verticalement, suggèrent cependant que la disposition primaire des pièces a été sensiblement modifiée par des phénomènes post-dépositionnels, vraisemblablement liés à un processus de retrait et de gonflement répété des argiles. Ce processus se marque à l'échelle du terrain par la présence de faces lisses et brillantes sur les agrégats argileux (slickensides), dont la formation résulte des pressions qui apparaissent dans le sol au cours des phases d'humectation et de gonflement. Une mesure de la fabrique a été faite de manière à quantifier ce phénomène. Les résultats montrent une disposition très désordonnée du grand axe des objets (fig. 11). Sur un diagramme de Benn, le point représentatif du niveau archéologique s'écarte sensiblement des valeurs obtenues pour des sites comme Etiolles et se positionne à proximité du pôle fabrique isotrope (fig. 12). Si l'existence de perturbations postdépositionnelles du site ne fait donc pas de doute, on pense toutefois que celles-ci n'ont pas oblitéré de manière significative l'organisation spatiale originelle du niveau, dans la mesure où leur impact reste très local. En revanche, elles expliquent probablement en grande partie la dispersion verticale des objets, qui dépasse $30 \mathrm{~cm}$. 
Fig. 11 - Fabriques relevées sur le site de Croix-de-Canard, diagrammes de Schmidt Fig. 11 - Fabrics of Croix-de-Canard site, Schmidt diagrams

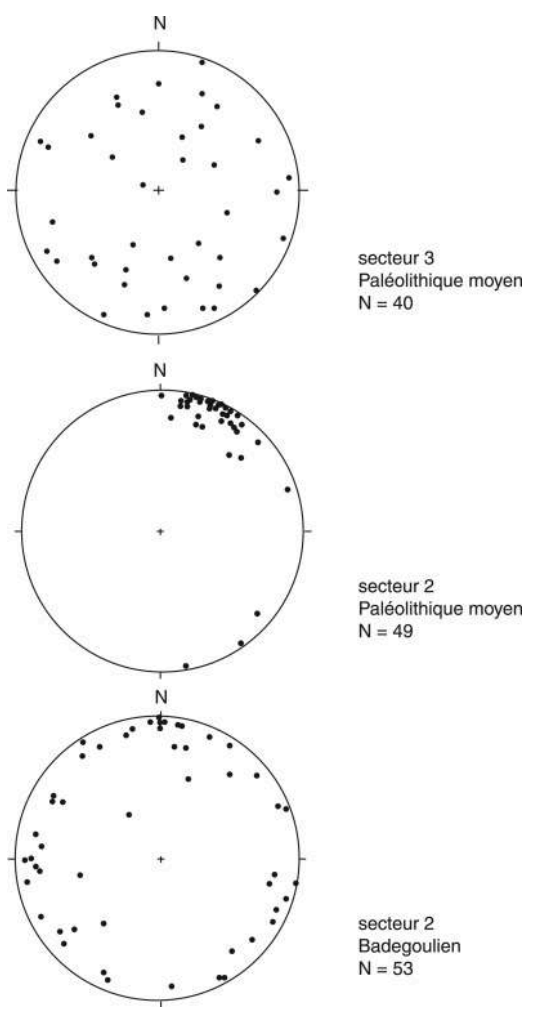

Le second niveau (secteur 2) est inclus dans des sédiments mal triés, interprétés comme des colluvions. Les différents éléments stratigraphiques et chronologiques récoltés indiquent que les dépôts dans lesquels ont été trouvés les objets archéologiques nappent la surface d'un cône de déjection qui a été abandonné par les écoulements torrentiels au cours d'une phase ancienne de l'évolution du versant. Pendant le Pléistocène récent, cette surface n'a reçu que de très rares apports sédimentaires et les vestiges archéologiques n'ont pas été profondément enfouis. Un tel type de contexte a plusieurs implications en ce qui concerne l'archéologie :

- des niveaux d'occupation d'âge différent peuvent être situés à des profondeurs voisines ; il est difficile d'établir une stratigraphie relative des occupations successives;

- dans la mesure où les vestiges sont restés exposés en (sub-)surface pendant une longue période, les phénomènes de remaniement par les processus naturels postérieurs à l'abandon du site par les hommes peuvent avoir joué un rôle important dans la constitution des niveaux archéologiques ; le contexte est donc peu favorable à une bonne préservation des niveaux d'occupation.

De manière à tester cette hypothèse, les fabriques de différents locus au sein de la nappe de vestiges ont été analysées. Les résultats obtenus montrent sans ambiguïté la présence d'une orientation préférentielle très marquée des objets selon la pente (fig. 11). Une telle orientation préférentielle n'a jamais été observée dans des sites archéologiques non perturbés ; en revanche, la comparaison avec les fabriques relevées dans différents types de dépôts de pente naturels indique que les valeurs trouvées entrent dans le champ de celles connues pour la solifluxion (fig. 12), c'est-à-dire de la reptation du sol liée aux alternances de gel et de dégel en contexte périglaciaire. Il apparaît donc très vraisemblable que le niveau paléolithique moyen ait été remanié par la dynamique de versant au cours des phases froides du Pléistocène récent. Dans l'état 
actuel des connaissances, l'intensité du déplacement des objets reste difficile à apprécier sur la seule base de la fabrique. L'absence de véritable concentration et la faible densité des vestiges plaident en faveur d'un remaniement important et d'une dispersion sur une grande surface d'unités initialement mieux délimitées. On peut à ce sujet comparer le plan de répartition du secteur 2 (locus 3), où les objets forment une nappe à peu près continue, avec celui du secteur 3 évoqué plus haut, pour lequel l'étude taphonomique ne met en évidence aucun déplacement horizontal significatif et où des amas très denses séparés par des zones quasiment stériles apparaissent clairement (fig. 13). Ce phénomène de dispersion est en accord avec les données recueillies sur des coulées de solifluxion actuelles (Texier et al. 1998), qui indiquent à la fois une translation vers l'aval et une " diffusion " du matériel expérimental déposé à la surface des coulées. L'hypothèse d'un remaniement important du niveau archéologique par la dynamique de versant en milieu périglaciaire rend donc bien compte des différentes observations effectuées sur le site. En raison du remaniement, toute lecture palethnologique de l'organisation spatiale des vestiges semble incertaine, au moins à l'échelle de surfaces restreintes; pour autant, il n'implique pas obligatoirement une perte de l'intégrité de l'assemblage lithique initial. En effet, dans la mesure où d'une part la solifluxion n'entraîne pas de tri longitudinal important des objets au cours des mouvements sur la pente, qu'il s'agit d'autre part d'un site de plein air dans lequel il est peu vraisemblable que de nombreuses occupations se soient succédées au même endroit (des mélanges d'industries sont donc improbables) et qu'enfin, la fouille a concerné une superficie importante (de 100 à $250 \mathrm{~m} 2$ pour chaque locus), on peut considérer que la plus grande partie du matériel archéologique qui composait le site originel nous soit parvenu. C'est ce que tendent à démontrer la présence de nombreux remontages au sein du niveau étudié. Ainsi, le taux de remontage atteint $37 \%$ du total du matériel récolté sur le locus 3 , valeur comparable à celle trouvée dans de nombreux sites d'habitats paléolithiques. 
Fig. 12 - Fabriques du site de Croix-de-Canard, diagramme de Benn Fig. 12 - Fabrics of Croix-de-Canard site, Benn's diagram

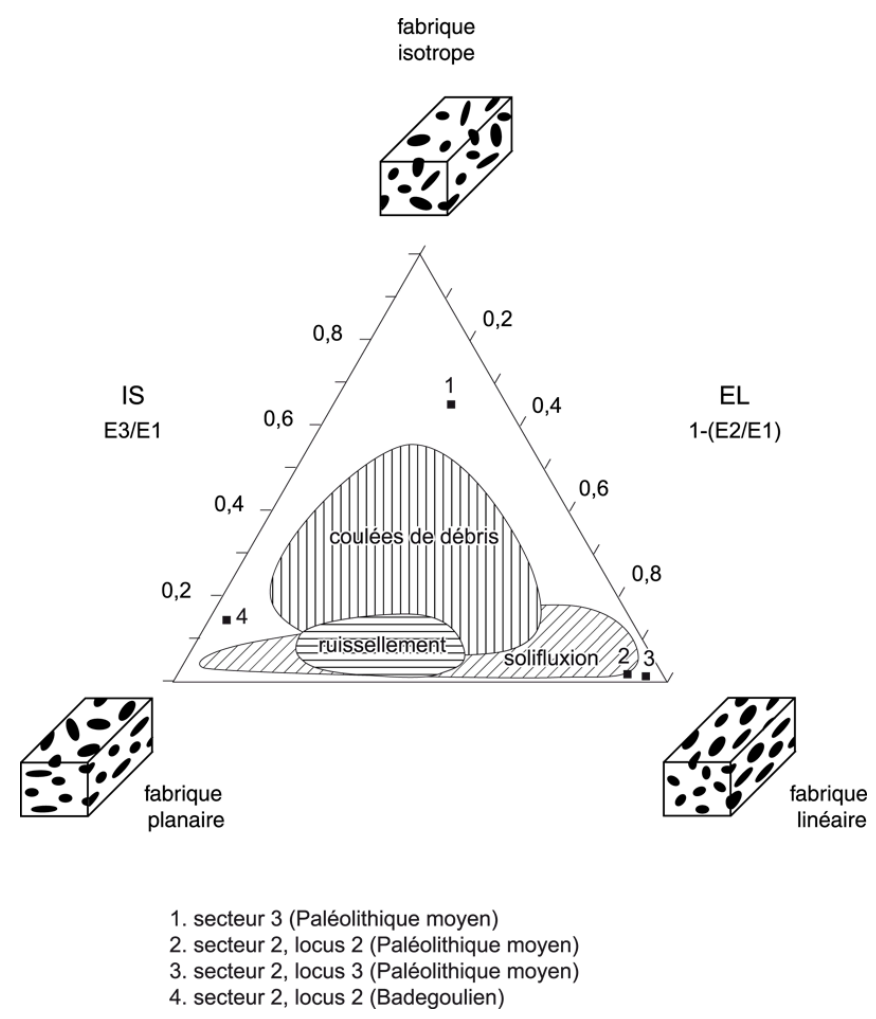

Les aires correspondant à différents processus naturels sont indiquées en hachures The areas that correspond to naturals processes are hatched 
Fig. 13 - Plans de répartition des vestiges sur le site de Croix-de-Canard Fig. 13 - Map of artefact distribution at Croix-de-Canard

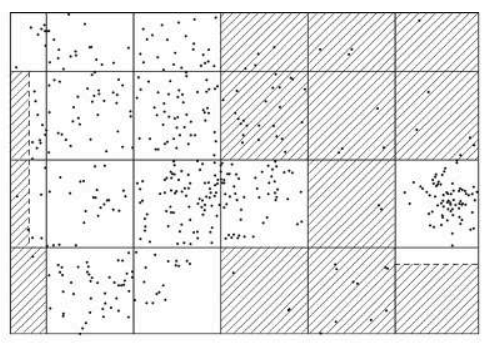

$\square$ fouille manuelle $\quad$ secteur 2 , locus 3

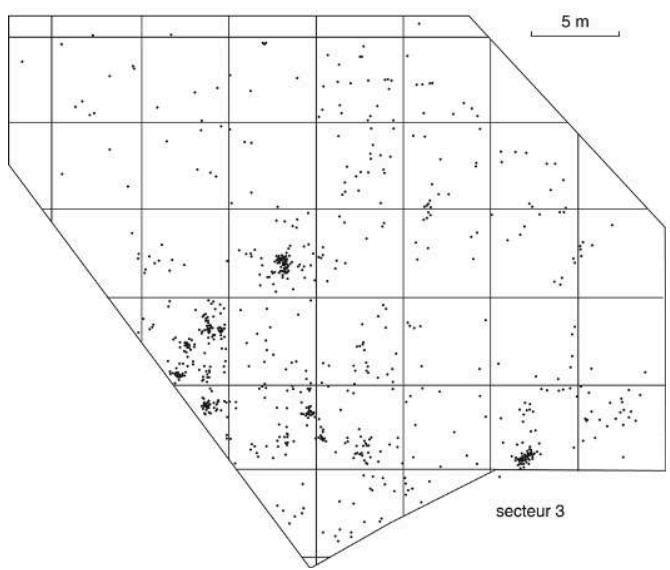

D’après L. Detrain (Document Final de Synthèse, rapport non publié, INRAP Grand Sud-Ouest, 2002) From L. Detrain (Document Final de synthèse, unpublished report, INRAP Grand Sud-Ouest, 2002)

\section{Caminade}

Le site de Caminade est un vaste abri-sous-roche du Périgord (fig. 9) qui a été fouillé de 1953 à 1968 par B. Mortureux et D. de Sonneville-Bordes. La partie est du gisement a particulièrement retenu l'attention des fouilleurs qui y ont reconnu une séquence archéologique constituée de trois niveaux moustériens surmontés de deux niveaux d'Aurignacien ancien puis de deux niveaux d'Aurignacien récent (Sonneville-Bordes, 1969, 1970). Une recherche systématique de raccords entre les différents assemblages lithiques isolés lors de fouilles anciennes a montré l'impossibilité de séparer les deux niveaux d'Aurignacien ancien d'une part et les deux niveaux d'Aurignacien récent d'autre part (Bordes, 2000 ; Bordes et Lenoble, 2001). Une reprise des travaux a alors été entreprise pour discuter les processus de formation du site.

Les niveaux aurignaciens sont interstratifiés dans des sables argileux massifs, interprétés comme des dépôts ruisselés à l'aval d'un cône colluvial qui pénètre dans l'abri sous roche. Le nombre élevé de vestiges mesurés $(n=171)$ dans la nappe archéologique supérieure (Aurignacien récent) permet d'observer en détail l'évolution de la fabrique en fonction de la stratigraphie. Le calcul des paramètres $L$ et $\mathrm{p}$ sur des séries de 40 objets se succédant dans l'ordre stratigraphique met en évidence des variations importantes (fig. 14) : la fabrique est de type planaire à la base et au sommet de la nappe de vestiges, tandis qu'une orientation préférentielle apparait dans la zone médiane. Ces variations démontrent que la constitution de la nappe, d'apparence homogène si l'on en juge d'après les projections verticales, ne correspond en réalité pas 
à un événement simple mais résulte d'une succession complexe de processus anthropiques et naturels.

Fig. 14 - Site de Caminade : évolution verticale de la valeur de $L$ dans la nappe de vestiges de l'Aurignacien récent

Fig. 14 - Caminade: vertical evolution of $L$ value within the upper Aurignacian artefact level
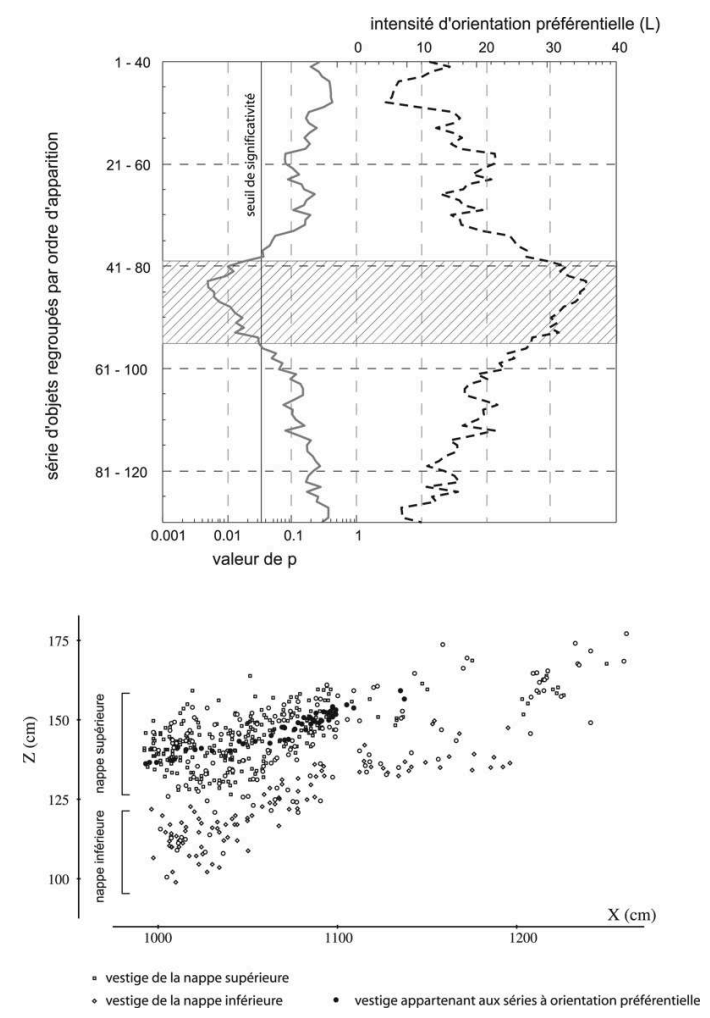

\section{Synthèse des analyses en milieu archéologique}

Au cours de la dernière décennie, de nombreux sites paléolithiques ont donné lieu à une étude contextuelle détaillée et d'abondantes données sur les fabriques ont été récoltées. Les figures 15 et 16 présentent les valeurs tirées de la base de données établie par P. Bertran, A. Lenoble et J.P. Texier (IPGQ, Talence). La lecture des diagrammes met en évidence les points suivants :

1) pour les sites pour lesquels aucune présomption de remaniement n'existe, comme Etiolles (amas sur pente faible ou nulle) ou Hope Estate (site précolombien des Petites Antilles), de même que pour les sites expérimentaux, les valeurs du paramètre $\mathrm{r} 1$ obtenu avec la Méthode des Valeurs Propres sont comprises entre 0 et 0,45 tandis que les valeurs de $\mathrm{r} 2$ sont entre 2 et 4 . Sur un diagramme de Benn, les points représentatifs se placent à proximité du pôle fabrique planaire. L'aire définie par ces sites englobe également les valeurs obtenues au cours de l'expérience de Lenoble et Bordes (2001) sur des niveaux piétinés lorsque la pente est inférieure à $10^{\circ}$;

2) les sites inclus dans des dépôts soumis à des pédoturbations (retrait-gonflement des argiles, action de la flore et de la faune du sol) ont des fabriques plus désordonnées ; sur un diagramme de Benn, leurs points représentatifs s'étirent en direction du pôle IS = 1 (fabrique isotrope). Ces mécanismes sont supposés n'avoir qu'un impact restreint sur la 
configuration spatiale initiale du niveau archéologique. La stratification peut être en revanche très perturbée ;

3) l'action du ruissellement, si elle peut avoir de grandes conséquences sur la répartition spatiale des vestiges, ne modifie pas nécessairement la fabrique. Les différentes données obtenues montrent un grand étalement des valeurs à la fois en direction du pôle IS $=1$ et du pôle $\mathrm{EL}=1$; les fabriques restent néanmoins plus ou moins nettement planaires. Les raisons de l'augmentation de l'isotropie restent en partie conjecturales; il est probable que dans de nombreux cas, d'autres mécanismes que le ruissellement, notamment des pédoturbations, soient intervenus dans l'enfouissement des niveaux analysés. Les valeurs de IS les plus basses (ou de r2 les plus élevées) proviennent du site badegoulien de Marsas (fouilles inédites I. Ortega, INRAP), dans la région de Blaye en Gironde, qui est inclus dans des sables éoliens repris par le ruissellement. On pense que de telles valeurs qui indiquent une position très à plat des vestiges (plus à plat que dans un site comme Etiolles) résultent d'un phénomène de résidualisation du niveau archéologique sur le versant par le ruissellement ;

4) les phénomènes de transport en masse des vestiges, généralement par solifluxion pour les sites analysés, se traduisent par l'apparition d'une orientation préférentielle dans la pente, laquelle peut être faible et ne pas dépasser 3 à $4^{\circ}$. Sur un diagramme de Benn, tous les sites provenant de dépôts soliflués s'isolent très bien des autres niveaux analysés et se placent en direction du pôle $\mathrm{EL}=1$ (fabrique linéaire). Ils correspondent à des niveaux du Paléolithique moyen et du Paléolithique supérieur ancien, c'est-à-dire à des niveaux dont la mise en place a précédé ou a été contemporaine des périodes les plus froides du Pléistocène. Dans notre échantillon, ce type de fabrique n'a en revanche pas été rencontré dans les sites postérieurs au dernier Maximum Glaciaire (Badegoulien, Magdalénien, Epipaléolithique, Mésolithique). Selon les cas, ce type de transport peut avoir occasionné des perturbations plus ou moins significatives des niveaux d'occupation. 
Fig. 15 - Fabrique des différents sites archéologiques analysés, diagramme de Benn Fig. 15 - Fabric of the study sites, Benn's diagram

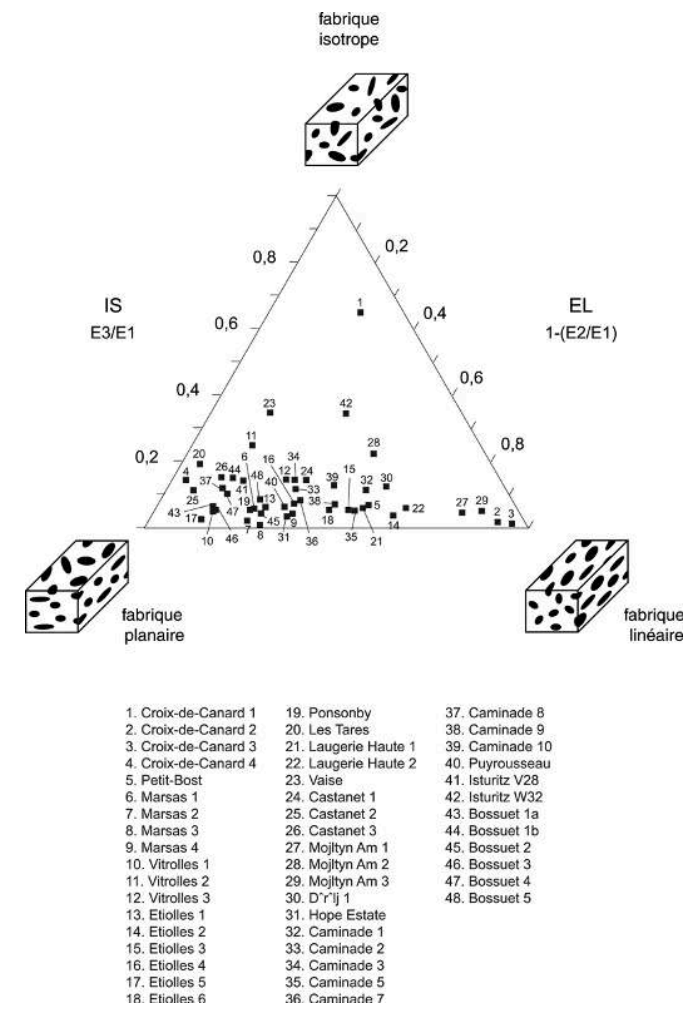

Fig. 16 - Fabriques et types de contexte sédimentaire des sites. Les regroupements sont effectués en tenant compte des principaux processus de perturbation déduits des études géoarchéologiques Fig. 16 - Fabric and sedimentary context of the sites. Types of disturbances are assumed from geoarchaeological studies

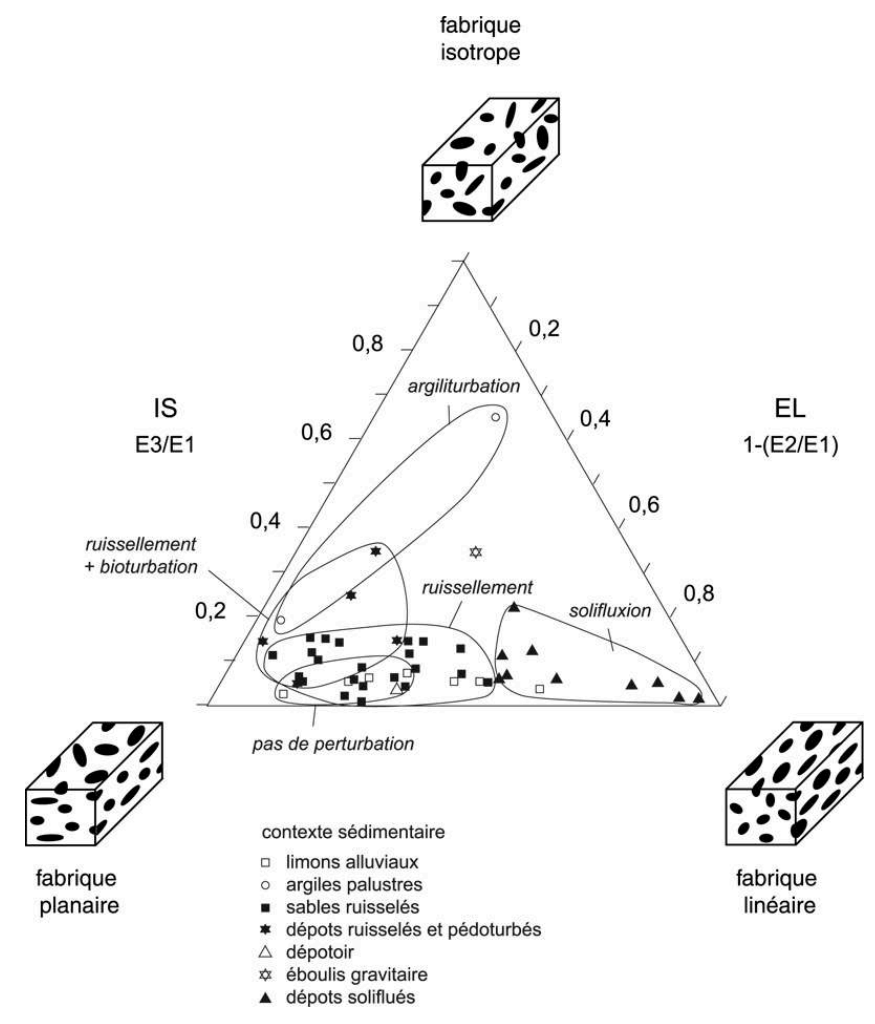




\section{Conclusion}

Bien que de nombreuses questions comme celle soulevée par l'analyse du site d'Etiolles restent encore en suspens et nécessitent que de nouveaux travaux leur soient consacrés, les progrès réalisés au cours de la dernière décennie dans l'analyse des fabriques, concernant l'exploitation statistique des mesures mais aussi la constitution d'un référentiel sur les différents mécanismes sédimentaires, en font une méthode très efficiente pour aborder le mode de constitution des niveaux archéologiques. Elle permet notamment de discriminer clairement les niveaux ayant subi des pédoturbations importantes ou un transport en masse par la solifluxion, cas fréquemment rencontré dans les sites paléolithiques européens contemporains de climats périglaciaires. Cependant, si elle a le mérite d'attirer l'attention sur un ou plusieurs mécanismes perturbateurs, cette méthode ne permet pas seule d'apporter des éléments précis sur le degré de remaniement ou sur l'intégrité d'un assemblage lithique. Elle doit pour cela être confrontée aux autres données géologiques et archéologiques, provenant de l'étude des remontages, de l'état de surface des pièces, de l'homogénéité des chaînes opératoires ou des assemblages d'outils. Le cas du site de Croix-de-Canard développé plus haut montre par exemple comment le croisement des différentes approches peut permettre de cerner assez précisément les processus de formation d'un niveau archéologique et de poser des limites à l'interprétation archéologique de l'assemblage lithique récolté. On peut souligner que pour le niveau moustérien du secteur 2 de ce site, les différentes données montrent qu'en dépit d'un remaniement important par des processus périglaciaires, l'homogénéité de l'assemblage lithique peut être admise avec un degré de confiance élevé. C'est vraisemblablement le cas pour de nombreux sites de plein air, dans lesquels les différentes occupations sont bien séparées en stratigraphie et dans l'espace. En revanche, c'est rarement le cas pour les sites en grotte ou en abri sous roche, dans lesquels de nombreuses phases d'occupation se sont succédées sur un même espace à des intervalles de temps relativement brefs et où activité anthropique et phénomènes géologiques postdépositionnels ont donné naissance à des palimpsestes difficilement déchiffrables. Peu d'études taphonomiques détaillées sont cependant disponibles pour ces sites, qui ont pour la majorité d'entre eux fait l'objet de fouilles anciennes. L'analyse des fabriques, que l'on peut pratiquer sur les objets visibles en coupe, peut constituer un moyen rapide pour proposer une première réévaluation de la signification des assemblages lithiques provenant de ces sites, eu égard à leur importance dans la définition des différentes cultures du Paléolithique.

\section{BIBLIOGRAPHIE}

BAR YOSEF O. et TCHERNOV E., 1972 - On the palaeo-ecological history of the site of Ubeidiya, Israel.

Publications of the Israel Academy of Sciences and Humanities, 35 
BENN D.I., 1994 - Fabric shape and the interpretation of sedimentary fabric data. Journal of Sedimentary Research, A64, 4 : 910-915

BERTRAN P., 1994 - Dégradation des niveaux d'occupation paléolithiques en contexte périglaciaire : exemples et implications archéologiques. Paleo, 6: 285-302

BERTRAN P. et TEXIER J.P., 1995 - Fabric analysis: application to palaeolithic sites. Journal of Archaeological Science, 22: 521-535

BERTRAN P., HETU B., TEXIER J.P. et VAN STEIJN H., 1997 - Fabric characteristics of slope deposits. Sedimentology, $44: 1-16$

BERTRAN P. et TEXIER J.-P., 1997 - Géoarchéologie des versants. Les dépôts de pente. Dynamique du paysage. Entretiens de géoarchéologie, J.-P. Bravard et M. Prestreau (eds.), Documents d'Archéologie en Rhône-Alpes, 15 : 59-86.

BORDES J.-G., 2000 - La séquence aurignacienne de Caminade revisitée : l'apport des raccords d'intérêt stratigraphique. Paleo, $12: 387-407$

BORDES J.-G. et LENOBLE A., 2001 - Caminade. Rapport final de synthèse de fouille programmée, Service Régional de l'Archéologie d'Aquitaine, Bordeaux

BUTZER K. W. 1982 - Archaelogy as human ecology. Cambridge University Press, 364 p.

CURRAY, J. R. 1956 - Analysis of two-dimensional orientation data. Journal of Geology, 64 : 117-134.

HENRY J., 1976 - Manuel d'analyses structurales. Méthodes modernes de géologie de terrain, Technip, Paris.

ISAAC G.L., 1967 - Towards the interpretation of occupation debris: some experiments and observations. Kroeber Anthropological Society Papers, 37: 31-57

KAUFULU Z. M. 1987 - Formation and preservation of some earlier stone age sites at Koobi Fora, Northern Kenya. South African Archaeological Bulletin, 42 : 23-33

LENOBLE A. et BORDES J.G., 2001 - Une expérience de piétinement et de résidualisation par ruissellement. Préhistoire et approche expérimentale, L. Bourgignon, I. Ortega et M.C. Frère-Sautot (eds.), coll. Préhistoire n 5, Monique Mergoil, Montagnac: 295-311

MAC EACHRAN D. B. 1990 - Stereo, the stereographic projection programme. Apple @ Macintosch Computer software, version 1.3

MARK D.A., 1973 - Analysis of axial orientation data, including till fabrics. Geological Society of America Bulletin, 84: 1369-1374

MILLS H.H., 1983 - Clast-fabric strength in hillslope colluvium as a function of slope angle. Geografiska Annaler, 65A: 255-262

SCHICK K.D., 1986 - Stone age sites in the making. Experiments in the formation and transformation of archaeological occurences. Bar international series $n^{\circ} 319,313 \mathrm{p}$.

SONNEVILLE-BORDES D. de, 1969 - Les industries moustériennes de l'abri Caminade est, commune de la Canéda (Dordogne). Bulletin de la Société Préhistorique Française, 66 : 293-310

SONNEVILLE-BORDES D. de, 1970 - Les industries aurignaciennes de l'abri Caminade est, commune de la Canéda (Dordogne). Quaternaria, XIII : 77-131

TABORIN Y. (dir.), 1994 - Environnements et habitats magdaléniens dans le centre du Bassin Parisien. Documents d'Archéologie Française, n 43, Maison des Sciences de l'Homme, Paris, 189 p. 
TEXIER J.P., BERTRAN P., COUTARD J.P., FRANCOU B., GABERT P., GUADELLI J.L., OZOUF J.C., PLISSON H., RAYNAL J.P. et VIVENT D., 1998 - An experimental archaeological program in periglacial environment : problem, methodology, first results. Geoarchaeology, 13, 5 : 433-473

TODISCO D., BERTRAN P. et PECH P., 2000 - Déplacements superficiels et contrôle climatique des coulées à front pierreux de La Mortice, Haute-Ubaye, Alpes françaises du Sud. Permafrost and Periglacial Processes, 11: 97-108

WATSON G.S., 1965 - Equatorial distributions on a sphere. Biometrika, 52: 193-201

WATSON G.S., 1966 - The statistics of orientation data. Journal of Geology, 74: 786-797

WOODCOCK, N. H. 1977 - Specification of fabric shapes using an eigenvalue method. Geological Society of America Bulletin, 88 : 1231-1236.

\section{RÉSUMÉS}

Des progrès significatifs ont été réalisés au cours de la dernière décennie dans l'analyse des fabriques en contexte archéologique, c'est-à-dire dans l'analyse de l'orientation et du pendage du grand axe des objets. Ils concernent à la fois l'exploitation statistique des mesures, la constitution d'un référentiel sur les mécanismes sédimentaires naturels ainsi que l'accroissement du nombre de sites étudiés. Ces travaux montrent que les fabriques constituent un outil efficace pour aborder les processus de formation des niveaux paléolithiques. Elles permettent notamment de discriminer clairement les niveaux ayant subi des pédoturbations importantes dues à l'activité biologique et au retrait-gonflement des argiles ou les niveaux affectés par la solifluxion en contexte périglaciaire. Dans l'état actuel des connaissances, les fabriques utilisées seules ne permettent pas d'estimer réellement l'ampleur des perturbations et doivent pour cela être confrontées avec d'autres arguments archéologiques et géologiques.

Fabric analysis in archaeological contexts, i.e. the study of the strike and dip of the artefacts, has been significantly improved during the last decade, mainly with respect to the statistical treatment of data, the constitution of a referential for natural sedimentary processes as well as the increased number of data for palaeolithic levels. These works show that fabrics are an efficient tool for assessing site formation processes. Fabrics allow discrimination between i) undisturbed (or poorly disturbed) levels, ii) levels that have undergone strong pedoturbations due to biological activity or to striking and swelling of clayey sediments and iii) levels that have been significantly reworked by slope processes such as periglacial solifluction or overland flow. However, in the present state of knowledge, fabrics do not allow precise estimation of the degree of perturbation if not associated with other archaeological and geological criteria. From the set of data on French palaeolithic sites used here, it is assumed that slope dynamics and particularly solifluction for sites predating the older Dryas may have played an important role in site formation.

\section{INDEX}

Mots-clés : fabrique, taphonomie, processus de formation des sites archéologiques, Paléolithique, fabrics, taphonomy, site formation processes, Palaeolithic 


\section{AUTEURS}

\section{PASCAL BERTRAN}

INRAP, Centre d'activité Les Echoppes, 156 avenue Jean Jaurès, F-33600 Pessac et U.M.R. 5808

ARNAUD LENOBLE

IPGQ, U.M.R. 5808, bâtiment de géologie, avenue des facultés, F-33405 Talence 